\title{
Experimental studies of the adsorption of oxygen and nitric oxide at $\mathrm{Ni}(100)$
}

\author{
M Volkmer, K Nolting, G H Fecher, B Dierks and U Heinzmann, Fakultät für Physik, Universität \\ Bielefeld, D-4800 Bielefeld, FRG
}

This paper presents and discusses the adsorption of $\mathrm{O}_{2}$ and $\mathrm{NO}$ at $\mathrm{Ni}(100)$. The adsorption of $\mathrm{O}_{2}$ at $\mathrm{Ni}(100)$ is found to occur in four phases as indicated by LEED $(p(2 \times 2), c(2 \times 2), c(2 \times 2 / \sqrt{ } 3)$, NiO) and AES, whereas in the work function changes, only three dominant phases are evident. In the case of NO we found purely molecular adsorption at temperatures below $170 \mathrm{~K}$ in a disordered phase. At room temperature, molecular as well as dissociative adsorption (LEED c $(2 \times 2)$ ) occurs. NO is desorbed at $380 \mathrm{~K}$, but the LEED pattern is still visible even at higher temperatures up to $800 \mathrm{~K}$. The superstructure of NO at Ni(100) is a N-and O-superstructure. The measurement of $\Delta \varphi$ shows that the dipole moment and the polarizability of the adsorbed NO are markedly higher as compared to the data of the gas-phase molecule. No oxidation of the Ni(100) surface could be observed even at a very high (500 L) NO exposure.

\section{Introduction and experimental set-up}

We have studied the adsorption of $\mathrm{O}_{2}$ and $\mathrm{NO}$ at $\mathrm{Ni}(100)$. Although both systems have been the subject of several experimental studies, the oxidation of $\mathrm{Ni}(100)$ and the adsorption of $\mathrm{NO}$ at $\mathrm{Ni}(100)$ are still not completely understood ${ }^{1-8}$.

For precise studies of the oxidation process of $\mathrm{Ni}(100)$ and the adsorption of $\mathrm{NO}$ at $\mathrm{Ni}(100)$ we have used several experimental methods: low energy electron diffraction (LEED), Auger electron spectroscopy (AES), thermal desorption spectroscopy (TDS) and a modified self-compensation retarding field method for measurements of the change of the electron work function $(\Delta \varphi)$ $\left(\mathrm{SCRF}^{9}\right)$.

Furthermore, we have examined effects depending on the orientation of the molecules prior to the interaction with the surface $^{10-12}$.

The $\mathrm{Ni}(100)$ target is cleaned by $\mathrm{Ar}^{+}$-jon bombardment (1.5 keV, 4-8 $\mu \mathrm{Acm}^{-2}$; $30 \mathrm{~min}$ without heating and $30 \mathrm{~min}$ with heating at a temperature of $520 \mathrm{~K}$ ). The target is annealed in oxygen ( $1 \mathrm{~min}, 1 \times 10^{-7} \mathrm{mbar} \mathrm{O}_{2}$, at $T=710 \mathrm{~K}$ ) to remove carbon and carbon oxide, followed by reduction of residual oxygen by hydrogen ( $3 \mathrm{~min}, 2 \times 10^{-7}$ mbar $\mathrm{H}_{2}$ ) during cooling down to $520 \mathrm{~K}$. After the heat treatment the crystal shows a clear $(1 \times 1)$ LEED pattern and no impurities were detectable by AES.

Results and discussion

The Ni(100) crystal was exposed to oxygen at room temperature and the adsorption was monitored by LEED. Up to an exposure of $A=20 \mathrm{~L}$ (at $\left.p=5 \times 10^{-8} \mathrm{mbar}_{2}\right)^{*}$ we found the $p(2 \times$ 2)-superstructure with a corresponding coverage of $\Theta=1 / 4 \dagger$. At higher exposures the $c(2 \times 2)$-superstructure $(\Theta=1 / 2)$ occurred, as reported by other authors ${ }^{1-3}$. For more than $400 \mathrm{~L}$ we observed the NiO-LEED pattern. Simultaneously, after an oxy-

\footnotetext{
* For oxygen we have $1 \mathrm{~L}=7.14 \times 10^{18}$ atoms $\mathrm{m}^{-2}$, and for nitric oxide, $1 \mathrm{~L}=3.69 \times 10^{18}$ molecules $\mathrm{m}^{-2}$, both at $T=300 \mathrm{~K}$.

$\dagger \Theta=1 \mathrm{ML}$ corresponds to one layer $\mathrm{Ni}(100)$ which is equal to $16.1 \times 10^{18}$ atoms m$^{-2}$.
}

gen exposure of $A \geqslant 300 \mathrm{~L}$ eight additional diffraction spots appeared at a radius equivalent to that of the $\mathrm{Ni}(1 \times 1)$-spots (see Figure 1). This indicates the same nearest neighbour distance for the adsorbate and the substrate.

This structure was mentioned in 1974 by Holloway and Hudson ${ }^{1}$. Without further explanation they claimed that it does
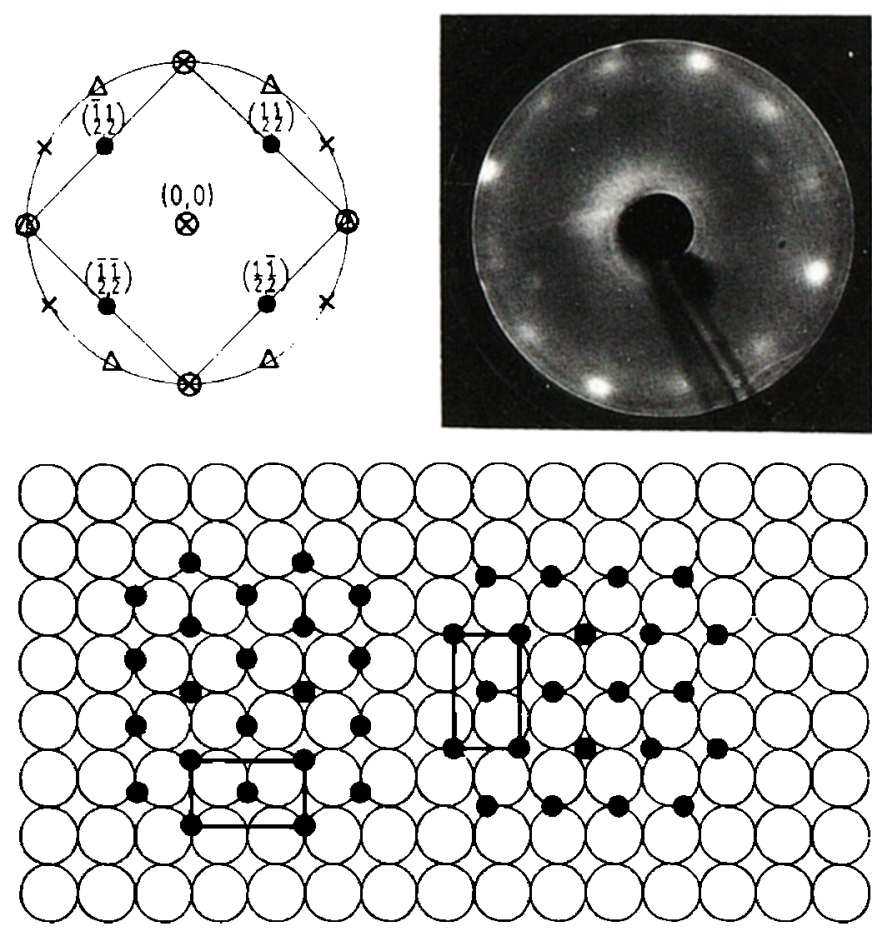

Figure 1. LEED-pattern of the simultaneously occurring $\mathrm{c}(2 \times 2)$ - and $c(2 \times 2 / \sqrt{3})$-structure; the symbols in the upper graph indicate: 0 . $\mathrm{Ni}(1 \times 1)$-spots; $x$, first domain; $\Delta$, second domain; $\mathrm{c}(2 \times 2)$-spots. In the lower part the schematic picture of the $c(2 \times 2 / / 3)$-structure is drawn, showing the two hexagonal domains rotated by $\pm 30^{\circ}$ with respect to the $\mathrm{Ni}(100)$-lattice. 

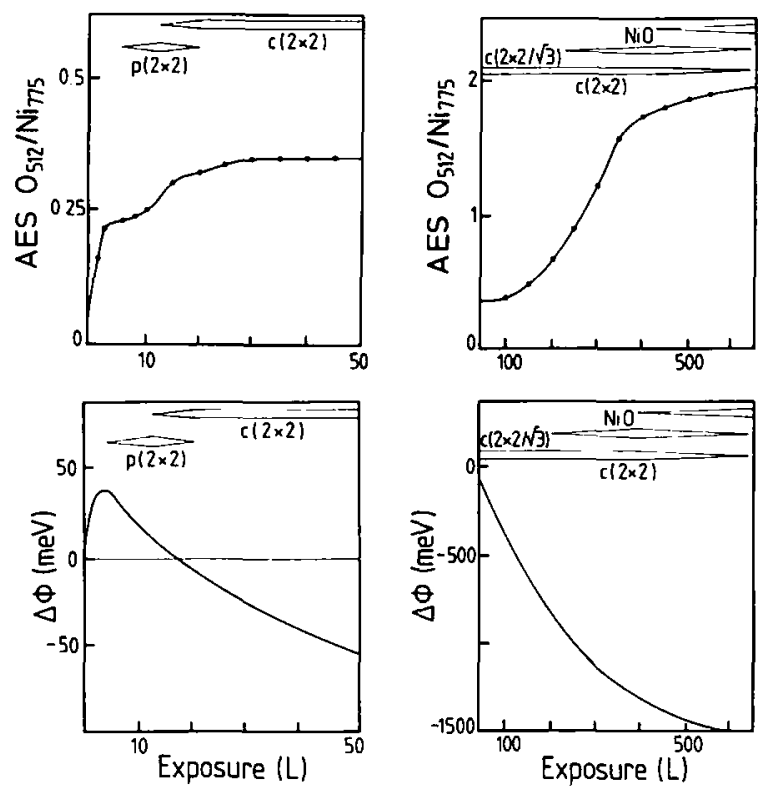

Figure 2. The ratio of the $\mathrm{O}_{512}$ to the $\mathrm{N}_{775}$ Auger amplitude and the change of the electronic work function as a function of oxygen exposure at $T=300 \mathrm{~K}$; left: low-exposure regime, right: high-exposure regime. Full drawn lines in the upper figures correspond to a calculation using a interpolating spline algorithm. The work function change was measured continuously during NO exposure.

not belong to a NiO-structure. Fargues and Ehrhardt ${ }^{13}$ and de Bokx et $\mathrm{al}^{14}$ have studied this twelve-spot pattern recently and explained it as two different domains of $\mathrm{NiO}(111)$ rotated by $\pm 30^{\circ}$ with respect to the $\mathrm{Ni}(100)$ surface.

We explain these additional spnts as being caused by a hexagonal structure of the oxygen overlayer. This structure is formed by compressing the $\mathrm{c}(2 \times 2)$-superstructure in one direction of the crystal surface by a factor of $\sqrt{3}$, while the distance in the perpendicular direction stays constant. This results in two hexagonal domains rotated by $\pm 30^{\circ}$ compared to the $\mathrm{Ni}(100)$ lattice (see Figure 1), where the oxygen atoms are in pseudobridge sites. The notation of this superstructure is given by $c(2 \times 2 / \sqrt{ } 3)$ with a corresponding coverage of $\Theta=1 / 2 \times \sqrt{3}$. The three oxygen-induced structures $c(2 \times 2), c(2 \times 2 / \sqrt{ } 3)$ and $\mathrm{NiO}(100)$ occurred simultaneously at an oxygen exposure between $400-600 \mathrm{~L}$.

Complementarily, we measured the $\mathrm{O}_{512}$ Auger amplitude* and the change of the work function for the system $\mathrm{O}_{2}-\mathrm{Ni}(100)$. In Figure 2 the ratio of the amplitudes $\mathrm{O}_{512}$ to $\mathrm{Ni}_{775}$ is shown as a function of the oxygen exposure. The ratio of the amplitudes increased rapidly up to $A=4 \mathrm{~L}$ and then stayed constant up to $A=10 \mathrm{~L}$. In this range of exposure we observed the $\mathrm{p}(2 \times$ $2)$-superstructure. When the $\mathrm{c}(2 \times 2)$-superstructure appeared the ratio of the Auger amplitudes increased slightly until the spots were completely developed. Then the ratio stayed constant followed by an increase at an oxygen exposure of above $A=$ $100 \mathrm{~L}$. In this range we observed the development of the $\mathrm{c}(2 \times$ $2 / \sqrt{ } 3)$-structure in addition to the spots of the $c(2 \times 2)$-structure and the NiO-structure. For an oxygen exposure of 400-500 L the

*We use the lower indices to indicate the energy of the Auger Transition. ratio of the Auger amplitudes reached saturation; the crystal was completely oxidized at the surface ( 2 or 3 layers). The diffraction pattern of the $\mathrm{c}(2 \times 2)$ - and the $\mathrm{c}(2 \times 2 / \sqrt{ } 3)$-superstructure were not observable any more. At an exposure above $A=700 \mathrm{~L}$ only the $\mathrm{NiO}(100)$-structure was visible. This is a reason why we favour the explanation that the $c(2 \times 2 / \sqrt{ } 3)$-superstructure is a chemisorbed phase of oxygen at $\mathrm{Ni}(100)$.

In Figure 2 the work function change $\Delta \varphi$ is shown as a function of the oxygen exposure. By measurements of the work function change we could only distinguish three phases of the oxygen adsorption. Up to an exposure of $A=4 \mathrm{~L}$ the work function increased by about $\Delta \varphi=40 \mathrm{meV}$ and the $\mathrm{p}(2 \times$ 2)-structure was observed. The positive initial change belongs to a chemisorption of the oxygen. When the $c(2 \times 2)$-structure became visible the work function turned negative. This indicated that the crystal had begun to oxidize. At an exposure $A \geqslant 700 \mathrm{~L}$ the work function change reached saturation.

The very high value of the change in the work function of $\Delta \varphi=-1.5 \mathrm{eV}$ is remarkable. The workfunction of $\mathrm{Ni}(100)$ is given by $\varphi=5.22 \mathrm{eV}^{15}$, so we get a value of $\varphi_{\mathrm{NiO}}=3.72 \mathrm{eV}$ for nickel oxide grown at $\mathrm{Ni}(100)$.

In further studies, $\mathrm{Ni}(100)$ was exposed to $\mathrm{NO}$ at room temperature ( $p=5 \times 10^{-8}$ mbar NO, 200s). The resulting diffraction pattern was a $c(2 \times 2)$-structure. The onset of the LEED-pattern at an exposure of $A=1 \mathrm{~L}$ indicates an initial sticking probability near unity. Subsequently, we studied the thermal desorption of NO. We observed one NO desorption peak at a temperature of $T=380 \mathrm{~K}$. This corresponds to a binding energy of approximately $1 \mathrm{eV}$, in agreement with Hamza et $a l^{16}$, the TDS spectra starting at $T=115 \mathrm{~K}$ showed the same behaviour as those starting at room temperature with cnly one NO desorption peak at $T=380 \mathrm{~K}$. We did not observe a second desorption peak indicating a second more weakly bound layer as found by Peebles et $a l^{8}$. When the NO was being desorbed the $c(2 \times 2)$-superstructure was still visible and the Auger spectra showed that there were both nitrogen and oxygen on the surface. The superstructure cannot only be due to the oxygen alone, because the maximum intensity of the diffraction pattern occurred at an energy $5 \mathrm{eV}$ lower than the energy at which the spots of the $c(2 \times 2)$-oxygen structure were observed most clearly. With TDS we observed the desorption of $\mathrm{N}_{2}$ at a temperature of $T=690 \mathrm{~K}$ corresponding to a binding energy of $1.6 \mathrm{eV}$. As in the case of NO-desorption the diffraction pattern did not change. We did not take $\mathrm{N}_{2}$-desorption spectra above temperatures $T \geqslant 750 \mathrm{~K}$ as Peebles $e t a l^{8}$ did, because at these temperatures carbon segregates from the bulk to the surface distorting TDS-measurement. The AES spectra still showed nitrogen which hints that a superstructure built by a simultaneous atomic chemisorption of nitrogen and oxygen occurs. This is in agreement with the predictions made by Passler et $a l^{4}$ from their SIMS measurements. A mixed $\mathrm{N}_{\mathrm{ad}}+\mathrm{O}_{\text {ad }}$ layer was also observed by Reimer et al ${ }^{17}$. For the system NO-Ni(100) we assume that dissociative adsorption of $\mathrm{NO}$ at room temperature takes place with simultaneous nitration which prevents oxidation. By TDS we were able to detect the presence of NO already molecularly desorbed at very low NO-exposures. This indicates a simultaneous molecular and dissociative chemisorption. We did not observe an oxidation of the $\mathrm{Ni}(100)$-surface even at extremely high NO-exposures $(A>500 \mathrm{~L})$ as Sakisaka et $a l^{6} \mathrm{did}$. They observed that above an exposure of $A=100 \mathrm{~L}$ the $\mathrm{Ni}(100)$ surface oxidized to $\mathrm{NiO}$ and the $\mathrm{O}_{512}$ Auger amplitude increased although the $\mathrm{N}_{379}$ Auger amplitude had reached saturation. We 


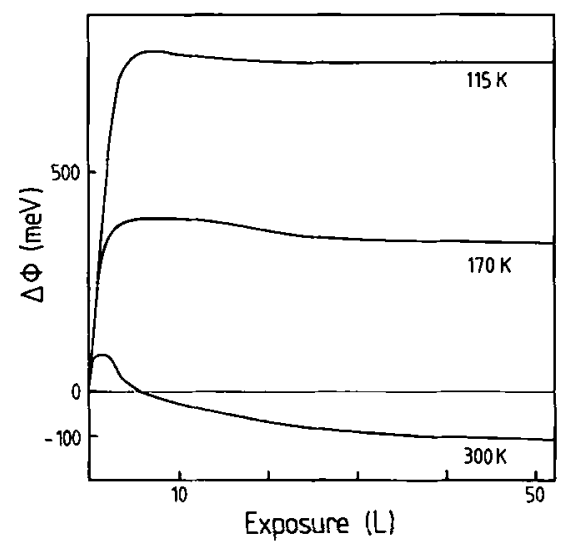

Figure 3. Continuously measured changes of the work function of NO at $\mathrm{Ni}(100)$ as function of $\mathrm{NO}$ exposure at different temperatures: $T=$ $115,170,300 \mathrm{~K}$

observed that the Auger amplitudes reach saturation at $A=50 \mathrm{~L}$ and remain unchanged at higher exposures.

Furthermore, the adsorption of NO was studied at temperatures between 115 and $170 \mathrm{~K}$. The change of the work function was monitored continuously during the NO-exposure (see $\mathrm{Fi}$ gure 3). The work function increased up to $A=4 \mathrm{~L}$ and reached saturation at higher exposures. The saturation value of the work function change was $\Delta \varphi=745 \mathrm{meV}$ at $T=115 \mathrm{~K}$ and $\Delta \varphi=$ $320 \mathrm{meV}$ at $T=170 \mathrm{~K}$. We observed no ordered superstructure for the adsorption of NO at low temperatures. This is in agreement with HREELS-measurements of Odörfer $e t a l^{18}$ and Avouris et $a l^{19}$ giving evidence that NO is adsorbed on Ni(100) on different surface sites. The superstructure already developed if the crystal was annealed up to temperatures above $T \geqslant 220 \mathrm{~K}$, where the onset of the $c(2 \times 2)$-structure occurs. This is in contrast to the observation by Price and Baker ${ }^{5}$ who reported this structure to occur at the NO desorption temperature of $T=380 \mathrm{~K}$. In this temperature range the dissociation of NO began. The curves of the work function change show distinctly the dissociative behaviour at a surface temperature of $T=300 \mathrm{~K}$ (see Figure 3).

The continuously measured work function changes show a deviation from linearity. Thus, the effective initial dipole moment $p_{0}$ and the effective polarizability $\alpha$ are calculated by fitting the coverage dependence of the change of the work function $\Delta \varphi$ to a Topping-model ${ }^{20}$ for a disordered adsorbate. For this fit we supposed an initial sticking probability of $S_{0}=1$ for $\mathrm{NO}$ at $\mathrm{Ni}(100)$ because the NO molecules have thermal, kinetic and rotational energies at room temperature (approximately $E_{\text {kin }}=$ $10 \mathrm{meV}$ ) without a preferred direction* and the surface is cooled to $T=115 \mathrm{~K}$. This is in agreement with our LEED and AES studies. The values obtained for the dipole moment $p_{0}$ and the polarizability $\alpha$ of adsorbed NO can be compared to the values of

* Note that the sticking probabilities measured in molecular beam experiments are lower, caused by the beam conditions.
NO in the gas phase ${ }^{21}$ :

$p_{0}=-0.25 \pm 0.01 \mathrm{D}$ (adsorbed); $|\mathrm{p}|=0.16 \mathrm{D}$ (gas phase)

and

$\alpha=2.6 \pm 0.3 \AA^{3}$ (adsorbed); $\alpha=1.7 \AA^{3}$ (gas phase).

The negative sign of the dipole moment shows that the NO= molecule is bond with the $\mathrm{N}$-end pointing to the $\mathrm{Ni}(100)$-surface. The increase of the dipole moment hints to a charge transfer from the substrate to the molecule. Peebles et $^{\mathrm{a}} \mathrm{l}^{\mathbf{8}}$ calculated the dipole moment to be $p=0.3 \mathrm{D}$, however they used a model that does not include molecule-molecule interactions.

It is remarkable that the initial slopes of the $\varphi$ vs exposure curves are the same at the different temperatures. This indicates that the initial sticking probability as well as the initial effective dipole moment are nearly equal independent of the temperature. Therefore we assume that the initial adsorption of $\mathrm{NO}$ at $\mathrm{Ni}(100)$ is molecular even at room temperature.

\section{Acknowledgements}

Financial support by the Deutsche Forschungsgemeinschaft (SFB 216, TP-P7) is gratefully acknowledged. The authors thank Dr N Böwering and Dr N Müller for valuable discussions and comments.

\section{References}

1 P H Holloway and J B Hudson, Surface Sci, 43, 123 (1974).

${ }^{2}$ T S Rahman, D L Mills, Y E Black, J M Szeftel, S Lehwald and H Ibach, Phys Rev, B30, 589 (1984).

${ }^{3}$ J E Demuth, N J DiNardo and G S Cargill, Phys Rev Lett, 50, 1373 (1983).

+ M A Passler, A Ignatiev, J A Schultz and J W Rabalais, Chem Phys Letl, 82, 198 (1981).

${ }^{5} \mathrm{G}$ L Price and B G Baker, Surface Sci, 91,571 (1980).

${ }^{6}$ Y Sakisaka, M Miyamura, Y Tamaki, M Nishijima and M Onchi, Surface Sci, 93, 327 (1980).

${ }^{7}$ M A Passler, T H Lin and A Ignatiev, J Vac Sci Technol, 18, 481 (1981).

${ }^{8}$ D E Peebles, E L Hardegree and J M White, Surface Sci, 148, 635 (1984).

${ }^{9} \mathrm{R}$ Nathan and B J Hopkins, J Phys Electron Sci Instrum, 7, 851 (1974).

${ }^{10} \mathrm{G}$ H Fecher, M Volkmer, B Pawlitzky, N Böwering and U Heinzmann, $J$ Chem Soc, Farad Trans, 85, (1989), in press.

${ }^{11} \mathrm{G}$ H Fecher, N Böwering, M Volkmer, B Pawlitzky and U Heinzmann Surface Sci, submitted.

${ }^{12} \mathrm{G}$ H Fecher, M Volkmer, B Pawlitzky, N Böwering and U Heinzmann, Vacuum, 41, 265 (1990).

${ }_{13}$ D Fargues and Y J Ehrhardt, Surface Sci, 209, 401 (1989).

${ }_{14}$ P K de Bokx, F Labohm, O L J Gijzeman, G A Bootsma and J W Geus, Appl Surface Sci, 5, 321 (1980).

15 J Hölzl and F K Schulte, In Solid Surface Physics, Springer Tracts in Modern Physics 85. Springer, Berlin (1979).

${ }^{16}$ A V Hamza, P M Ferm, F Budde and G Ertl, Surface Sci, 199, 13 (1988).

${ }^{17}$ W Reimer, Th Fink and J Küppers, Surface Sci, 193, 259 (1988).

${ }^{18} \mathrm{G}$ Odörfer, H-J Freund, G Watson, K D Tsuei and E W Plummer, Verhandlungen der DPG, abstract 0-15.3, Spring (1989).

${ }^{19} \mathrm{Ph}$ Avouris, N J DiNardo and J E Demuth, J Chem Phys, 80, 491 (1984).

${ }^{20} \mathrm{~J}$ Topping, Proc $R$ Soc, A114, 67 (1927).

${ }^{21}$ Handbook of Chemistry and Physics, 67th Edn. CRC Press, FL (1986-87). 